\title{
Mesoporous silica films with varying porous volume fraction: direct correlation between ortho-positronium annihilation decay and escape yield into vacuum
}

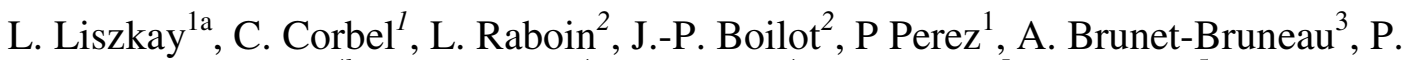 \\ Crivelli $^{4 b}$, U. Gendotti ${ }^{4}$, A. Rubbia ${ }^{4}$, T. Ohdaira ${ }^{5}$, R. Suzuki $^{5}$ \\ ${ }^{1}$ CEA, IRFU and IRAMIS, 91191 Gif-sur-Yvette Cedex, France \\ ${ }^{2}$ Laboratoire de Physique de la Matière Condensée, École Polytechnique-CNRS UMR \\ 7643, 91128 Palaiseau, France \\ ${ }^{3}$ INSP Institut des NanoSciences de Paris, Université Paris 6, UMR CNRS 7588, Campus \\ Boucicaut, 140 rue de Lourmel, 75015 Paris, France \\ ${ }^{4}$ Institut für Teilchenphysik, ETHZ, CH-8093 Zürich, Switzerland \\ ${ }^{5}$ AIST, Tsukuba, Ibaraki 305-8568, Japan
}

\begin{abstract}
The behavior of ortho-positronium (o-Ps) in mesoporous silica films implanted with lowenergy positrons has been studied as a function of the film porous volume fraction. A lifetime spectrometer allowed determination of $o$-Ps annihilation decay both inside and outside of the film. A kinetic model is introduced that permits the determination of the yield and rate of escape of $o$-Ps into vacuum as well as the annihilation decay rate of the trapped o-Ps in the film. It is shown that these undergo a sudden change at a threshold porous volume fraction, above which the o-Ps escape rate to vacuum varies linearly with volume fraction.

PACS numbers: 78.70.Bj, 61.43.Gt, 36.10.Dr, 78.20.Ci
\end{abstract}

\footnotetext{
a electronic mail: laszlo.liszkay@ @mki.kfki.hu; on leave from: KFKI Research Institute for Nuclear and Particle Physics, Budapest, Hungary.

${ }^{\mathrm{b}}$ present address: UFRJ, Rio de Janeiro, Brazil.
} 
Attention has recently been attracted to the experiments in fundamental physics $[1,2]$ that can be achieved once a high density, low energy ortho-positronium $(o-\mathrm{Ps})$ cloud is available. $O$ Ps is the triplet state of a positron-electron $\left(\mathrm{e}^{+}-\mathrm{e}^{-}\right)$bound pair. Its interaction with an antiproton beam provides a method for the production of neutral antihydrogen, suitable for direct gravity measurements of antimatter.

It has been early recognized that the $\mathrm{e}^{+}$lifetime spectra recorded with standard $\mathrm{e}^{+}$beam-based lifetime spectrometers give unreliable $o$-Ps lifetime distributions [3] in mesoporous films for which there is possible escape of $o$-Ps into vacuum [4]. Accordingly, to determine film porosity by analysis of $o$-Ps decay, various capping methods have been used to thwart this escape [4]. However, since the effect of capping on the mesoporous structures is unknown, it becomes questionable whether any information on pore volume distribution can be reliably extrapolated to virgin films. Additionally, the capping obscures any possibly interesting effects of film structure on the yield of $o$-Ps escape into vacuum.

In order to understand the $o$-Ps behaviour in films that generate free $o$-Ps into vacuum subsequent to positron implantation, accurate $o$-Ps lifetime distributions need to be recorded in the virgin films. The ETHZ slow positron beam-based lifetime spectrometer specifically designed to record $o$-Ps decay both inside and outside of a solid target is here used to study virgin mesoporous silica films. A surfactant templating method is applied to introduce mesopores and control their spatial organization, volume fraction and size distribution by varying the surfactant fraction. A kinetic model is introduced to describe the $o$-Ps escape into its vacuum state from its annihilation state in the film. Via analysis of the $o$-Ps decay times, the model permits a consistent separation between the yield of $o$-Ps remaining within the film and that which escapes into vacuum. It is established here that an $18 \%$ yield of escaped $o$-Ps suddenly develops at some threshold value of porosity at which there is also observed a large increase in the annihilation lifetime for the $o$-Ps remaining in the film. This increase is the fingerprint of a mesostructure reorganization that has previously escaped detection by other methods. The reorganization appears to not only trigger the $o$-Ps escape but to also reduce the annihilation rate of trapped $o$-Ps via electron pick-off. This correlation suggests either the onset of incoherent tunneling in the mesopore-well structure for $o$-Ps and/or the formation of a high fraction of open volume interconnections between the mesopores. Further work is in progress to establish the nature of the reorganization.

The mesoporous silica films are synthesised from mixtures containing the cetyl trimethyl ammonium bromide (CTAB) organic surfactant and a silica sol prepared by acidic hydrolysis of an ethanolic solution of tetraethoxysilane (TEOS) [5]. The total volume fraction occupied by the pores and the fraction of micropores $(<2 \mathrm{~nm})$ and mesopores $(\sim 2-50 \mathrm{~nm})$ is controlled in seven CTAB-TEOS $(\mathrm{S}(\mathrm{x}))$ Film/Glass $(\mathrm{S}(\mathrm{x}) / \mathrm{G})$

by varying the surfactant mole fraction, $\mathrm{CTAB} / \mathrm{Si}(x)$,from 0 to 0.14 . After deposition by spin coating and calcination at $450^{\circ} \mathrm{C}$, the thickness for the $\mathrm{S}(\mathrm{x})$ films determined from ellipsometry and profile measurements increases with $\mathrm{x}$ from 150 to $310 \mathrm{~nm}$. Due to the texture of the $\mathrm{S}(\mathrm{x})$ films, $\mathrm{X}$-ray patterns $(\lambda=1.54 \AA)$ consist of one intense peak at low angle $\left(2-4^{\circ}\right)$. For $x \geq 0.06$, the coherence length $\zeta$, i.e. the extent of the order in the thickness direction of a film, is deduced from the peak width at half maximum using the Scherrer formula. The values fall in the range 19-120 nm and are compared to the resolution of the diffractometer $(100-150 \mathrm{~nm})$ to classify the pore arrangement as a short or long range order (S/LRO). Consistent with the well organised Hexagonal 3D (H3D) structure for the pore 
arrangement in $\mathrm{S}(0.1)$ and the less organised Cubic (C) in $\mathrm{S}(0.14), \quad \zeta$ has a value $\geq 120 \mathrm{~nm}$ in $\mathrm{H} 3 \mathrm{D} \mathrm{S}(0.1)$ and $\sim 44 \mathrm{~nm}$ in $\mathrm{C} \mathrm{S}(0.14)$. The film porosity was determined in similar $\mathrm{S}(\mathrm{x})$ films deposited on silicium by in-situ coupling of ellipsometric porosimetry in the visible range with infrared ellipsometry (EP-IE) [6]. The total volume fraction occupied by micropores $\left(p_{\mu}\right)$ and mesopores $\left(p_{m}\right)$ is deduced from the changes of the refractive index in the film upon gas ethanol adsorption/desorption using for its value in the silica skeleton, 1.488. It increases from 0.33 to 0.58 for $0.06 \leq x \leq 0.14$. The relative populations changes with $x$ and reaches $\left(p_{\mu}, p_{m}\right) /\left(p_{\mu}+p_{m}\right)$ values of $(0.35,0.65)$ in $\mathrm{S}(0.10)$ and $(0.25,0.75)$ in $\mathrm{S}(0.14)$. Using the Kelvin equation and assuming spherical pores, a diameter of $4 \mathrm{~nm}$ has been determined for mesopores in $\mathrm{S}(0.10)$ films [6].

With the ETHZ $\mathrm{e}^{+}$beam [7]-based lifetime spectrometer, beam energy $\left(E_{e+}\right)$ was varied in the range 1-5 keV and the annihilation lifetime distribution recorded both inside and outside the film/glass [8-9]..Assuming a Makhovian stopping depth profile and no diffusion, calculations show that the $\mathrm{e}^{+}$stopping probability in a $\mathrm{S}(\mathrm{x})$ film of $1.5 \mathrm{gcm}^{-3}$ density and thickness, $\geq 250 \mathrm{~nm}$, is $\geq 92.5 \%$ for $E_{e+}=3 \mathrm{keV}$ and $\geq 46 \%$ for $E_{e+}=5 \mathrm{keV}$. The lifetime spectra that were recorded above $3 \mathrm{keV}$ are consequently a superposition of the lifetime spectra in the film and glass substrate.

For each $E_{e+}$ energy, the $\mathrm{e}^{+}$annihilation lifetime distribution is assumed to be a sum of exponential lifetime decay components, $\left(I_{i}, \tau_{i}\right)$. After background corrections and convolution with the 5ns FWHM resolution function of the spectrometer, the number of components resolved in the fitting of the spectra with the Lt9 program [10] is one for the $\mathrm{CTAB} / \mathrm{Si}$ mole fraction $x=0$ and three for $x \geq 0.03$. Given the $5 \mathrm{~ns}$ resolution, the shortest component, $\tau_{i=1}<<4 \mathrm{~ns}$, was disregarded due to the inaccuracy of its determination. Only the two longer ones, $\tau_{i=2,3}>\sim 10 \mathrm{~ns}$ (for any $x \geq 0.03$ ) were considered. Both depend on $E_{e+}$ and x. For $E_{e+} \geq 1 \mathrm{keV}$ and $x \leq 0.06$, the contribution of the longer component was too slight $\left(I_{3} \leq 2 \%\right.$ ) for reliable determination of $\tau_{3}$. On the other hand, for $E_{e+} \geq 1 \mathrm{keV}$ and $x \geq 0.08$, the longer component was well determined $\left(5 \leq I_{3} \leq 24 \%\right)$ and found always to decay with a lifetime of $\tau_{3}=142 \pm 5 \mathrm{~ns}$. This value is characteristic of the $o$-Ps self-annihilation lifetime in vacuum, $\tau_{v}=142 \mathrm{~ns}$. Accordingly, all lifetime spectra were fitted with $\tau_{3}$ constant and equal to $142 \mathrm{~ns}$ in order to obtain the remaining parameters of the fit, i.e., $I_{2}, I_{3}$, $\tau_{2}$, with better accuracy.

The energy dependence of these parameters shows that there are two regions in the films where the lifetime spectra differ: (i) a surface region and (ii) a homogeneous bulk region. For $E_{e+}<2 \mathrm{keV}$, the film surface contributes most importantly, and for $2 \leq E_{e+} \leq 3 \mathrm{keV}$, the film bulk dominates. For $E_{e+}>3 \mathrm{keV}$, the positron stopping in the glass substrate begins to contribute. Accordingly to make more reliable our analysis of the film bulk and avoid contributions to the decay curve from either the glass substrate or the film surface, all measurements reported here have used the ETHZ beam with $E_{e+}=3 \mathrm{keV}$ and, after film capping, the AIST beam with $E_{e+}=2 \mathrm{keV}$.

The existence in the decay curve of the $142 \mathrm{~ns}$ lifetime confirms, as earlier indicated [8], that the film bulk converts a fraction of the $\mathrm{e}^{+}$implanted in the film bulk into free $o$-Ps. For the 
remaining parameters of our fits, it should be noted that the dependences of $\tau_{2}$ (Fig. 1a) and $I_{2}$ (Fig. 1b) on $x$ tend to anticorrelate with that of $I_{3}$ (Fig. 1c). The steep increase in $I_{3}$ by an order of magnitude between $0.06 \leq x \leq 0.08$ marks the threshold for the escape of $o$-Ps into vacuum.

To interpret these results, a kinetic model is employed that describes the $o$-Ps escape process as a transition between two $o$-Ps states, both of which can independently decay via annihilation. Analogous models, so called "positron trapping" models, are widely used to calculate the $\mathrm{e}^{+}$lifetime distribution in presence of $\mathrm{e}^{+}$trapping into defects [11]. In the present model, the initial state is the film bulk $o$-Ps state characterized by the $o$-Ps annihilation lifetime, $\tau_{f}(\mathrm{x})$, for the $\mathrm{S}(\mathrm{x})$ film. The final state is the $o$-Ps ground state in vacuum characterized by the $\tau_{v}=142 \mathrm{~ns}$ annihilation lifetime. The transition probability per unit time from the film state to the vacuum state, i.e the escape rate of free $o$-Ps into vacuum, occurs at a rate $\kappa_{v}(x)$. For a given $x$, the occupancy probability of the film state, $n_{f}$, decreases via annihilation at the rate $\lambda_{f} n_{f}\left(\lambda_{f}=\tau_{f}^{-1}\right)$ and via escape to the vacuum state at the rate $\kappa_{v} n_{f}$. The occupancy probability of the vacuum state, $n_{v}$, decreases via annihilation at the rate $\lambda_{v} n_{v}\left(\lambda_{v}=\tau_{v}^{-1}\right)$ and increases at the rate $\kappa_{v} n_{f}$. The $o$-Ps lifetime distribution predicted by this model is then a sum of two exponentials, one with decay constant $\tau_{v}^{-1}$ and the other with a decay constant $\tau_{2}^{-1}=\lambda_{f}+\kappa_{v}$. By comparing the normalized experimental pre-exponential intensities $\left(i=2,3 \quad I_{i} /\left(I_{2}+I_{3}\right)\right.$ in Fig.1) with the pre-exponentials of the model, it is simple to deduce that the parameters of the model $\tau_{f}=\lambda_{f}^{-1}$ and $\kappa_{v}$ can be expressed in terms of the fitted parameters $\tau_{2}, \tau_{v}, I_{2}$, and $I_{3}$ as $\tau_{f}=\left(\left(\tau_{2}^{-1}-\tau_{v}^{-1}\right) I_{2} /\left(I_{2}+I_{3}\right)+\tau_{v}^{-1}\right)^{-1}$ and $\kappa_{v}=\left(\tau_{2}^{-1}-\tau_{v}^{-1}\right) I_{3} /\left(I_{2}+I_{3}\right)$. Also, with these, the yield of escaped o-Ps, $Y_{\nu}$, i.e. the annihilation probability in the vacuum renormalized to the total intensity, $I_{2}+I_{3}$, becomes simply $\mathrm{Y}_{v}=\left(I_{2}+I_{3}\right) \kappa_{v} /\left(\tau_{f}^{-1}+\kappa_{v}\right)$.

In Fig.1d-f, the values of $\tau_{f}, \kappa_{v}$ and $Y_{v}$ all appear to increase sharply at the value $x \approx 0.07$. In the case of porous solids, the quenching models for $o$-Ps annihilation by electron pick-off in solids indicate that the range 40-80 ns for the $\tau_{f}$ values (Fig. 1d) is consistent with $o$-Ps annihilation from localized states in mesopores of diameter greater than $3 \mathrm{~nm}[12,13]$. Furthermore, the steep increase in $\tau_{f}$ at $x \approx 0.07$ clearly implies an increase in the volume that localizes $o$-Ps. It can be inferred with a cubic pore model [13] that the mesopore size at $x \approx 0.07$ rapidly shifts from $\sim 4 \mathrm{~nm}$ to higher values of $\sim 5-6 \mathrm{~nm}$. Such a change in the mesopore size distribution however escapes detection by the other technique (EP-EI) used to estimate the pore size and for which the pore size is $\sim 4 \mathrm{~nm}$ over the range of $\mathrm{x}$ studied here. This suggests that $o$-Ps quenching is sensitive to some re-organisation of the mesopore distribution that is different from a simple increase in mesopore size. One possibility, consistent with the two type of pore size determinations, is that the re-organisation at $x \approx 0.07$ switches on incoherent o-Ps tunneling in the mesopore-well structure. Another is that the mesopores develop and interconnectivity at $x \approx 0.07$. Both phenomena are expected to also accommodate the sudden increase in the escape rate, $\kappa_{v}$ at $x \approx 0.07$ (Fig 1e) and corresponding increase in the yield of escaped $o$-Ps (Fig. 1f). According to EP-EI, the mesopore volume fraction at $x \approx 0.07$ then reaches $25 \%$, which corresponds to a mesopore 
density of about $10^{18} \mathrm{~cm}^{-3}$. It has been earlier suggested [14,15] that a sudden appearance of escaped o-Ps might serve as an indicator of a threshold for pore interconnection. Our results appear to confirm the need to examine this conjecture as one possibility.

In Fig. 2 we show that, above the $o$-Ps escape threshold at $x \approx 0.07$, the escape rate, $\kappa_{v}$, varies linearly with the mesopore volume fraction as determined from EP-EI. The slope, $0.18 \pm 1 \mathrm{~ns}^{-1}$ per unit of mesopore volume fraction, gives a determination for the $o$-Ps escape rate per mesopore with a 60-70 ns annihilation lifetime. The insert in Fig. 2 shows that, above its onset threshold, the $o$-Ps escape yield varies linearly as a function of the refractive index measured at $633 \mathrm{~nm}$ by spectroscopic ellipsometry.

To evaluate here the effect of capping on the pore size distribution, the $S(0.1)$ and $S(0.14)$ films were capped in AIST with a $20 \mathrm{~nm}$ thick silica layer in order to suppress $o$-Ps escape without, if possible, damaging the film mesostructure. For $E_{e+}=2 \mathrm{keV}$, the $270 \mathrm{ps}$ FWHM resolution of the AIST spectrometer allows us to resolve three lifetime components in capped $\mathrm{S}(0.1) / \mathrm{G}$ and four components in capped $\mathrm{S}(0.14) / \mathrm{G}$. For the longer components, the intensity and lifetime values after capping, $\left(I_{c i}, \tau_{c i}\right)$ are $(i=3 ; 25 \pm 3 \%, 67 \pm 2 \mathrm{~ns})$ for $x=0.1$ and $(i=3 ; 4.5 \pm 1 \%, 14 \pm 2 \mathrm{~ns} ; i=4 ; 19 \pm 1 \%, 87 \pm 2 \mathrm{~ns})$ for $x=0.14$. In $\mathrm{S}(0.1)$, the values before $\left(\tau_{f}\right)$ and after $\left(\tau_{c 3}\right)$ capping, are consistent at $67 \pm 2 \mathrm{~ns}$ in both cases. After capping in $\mathrm{S}(0.14)$, the average values of the two longer lifetimes, $\left(\left(I_{c 3} \tau_{c 3}\right)+\left(I_{c 4} \tau_{c 4}\right)\right) /\left(I_{c 3}+I_{c 4}\right)=72 \pm 2 \mathrm{~ns}$, agrees well with $\tau_{f}=70 \pm 1 \mathrm{~ns}$. Furthermore, the total intensity of the long components after capping is comparable to those before capping in both films: $23 \pm 2 \%$. This good agreement strongly supports the kinetic model used for data analysis before capping and indicates that silica capping does not appear to damage the mesostructure.

In summary, the present work provides a direct and reliable determination of the $o$-Ps yield and escape rate into vacuum for mesoporous films. Such a determination is crucial to the design of efficient low energy $\mathrm{e}^{+}$to free o-Ps mesoporous convertors for use in a new generation of antimatter experiments [1,2]. This work also provides the direct evidence that, at a mesopore density of about $10^{18} \mathrm{~cm}^{-3}, o$-Ps localization increases at the onset of $o$-Ps escape into vacuum implying either the existence of a threshold in pore density for o-Ps incoherent tunneling through the mesopore-well structure, and/or a threshold for the opening of pore interconnection. 


\section{References}

[1] P.Perez, A. Rosowsky, Nuclear Instrum. Meth. A 545, 20 (2005).

[2] A. Kellerbauer et al., Nucl. Instr. and Meth. B 266, 351 (2008).

[3] C. L. Wang, M. H. Weber, K. G. Lynn, K. P. Rodbell, Appl. Phys. Lett. 81, 4413 (2002).

[4] D. W. Gidley, W. E. Frieze, T. L. Dull, A. F. Yee, E. T. Ryan, H.-M. Ho, Phys. Rev. B 60, R5157 (1999).

[5] S. Besson, T. Gacoin, C. Ricolleau, C. Jacquiod, J-P. Boilot, J. Mater. Chem. 13, 404 (2003).

[6] A. Bourgeois, A. Brunet-Bruneau, S. Frisson, J. Rivory, M. Matheron, T. Gacoin, J-P. Boilot, Adsorption 11, 195 (2005).

[7] N. Alberola, T. Anthonioz, A. Badertscher, C. Bas, A.S. Belov, P. Crivelli, S.N. Gninenko, N.A. Golubev, M.M. Kirsanov, A. Rubbia, D. Sillou, Nuclear Instr. Meth. A 560, 224 (2006).

[8] L. Liszkay, C. Corbel, P Perez, P. Desgardin, M.-F. Barthe, T. Ohdaira, R. Suzuki, P. Crivelli, U. Gendotti, A. Rubbia, M. Etienne and A. Walcarius, Appl. Phys. Lett. 92, 063114 (2008).

[9] P. Crivelli, U. Gendotti, A. Rubbia, L. Liszkay, P.Perez, Mat. Sci. Forum 607, 251 (2009).

[10] J. Kansy, Nucl. Instrum. Meth. A 374, 235 (1996).

[11] P. Hautojärvi and C. Corbel, in: Positron Spectroscopy of Solids, Proceedings of the International School of Physics Course CXXV, edited by A. Dupasquier and A. P. Mills, IOS Press Amsterdam, Oxford, Tokyo, Washington DC, p. 491 (1995).

[12] T. Goworek, K. Ciesielski, B. Jasinska and J. Wawryszczuk Chem. Phys. Letters 272, 91-95 (1997).

[13] L. Dull, W. E. Frieze, D. W. Gidley, J. Sun, and A. F. Yee, J. Phys. Chem. B 105, 4657 (2001).

[14] M.P. Petkov, C.L. Wang, M.H. Weber, K.G. Lynn, K.P. Rodbell, J. Phys. Chem. 107, 2725 (2003).

[15] D. W. Gidley, H. G. Peng, and R. S. Vallery, Ann. Rev. Mater. Res. 36, 49 (2006). 
Figure Captions

Figure 1

Ortho-positronium measured lifetime components (left), $\left(\tau_{2}(a), I_{2}(b)\right)\left(142 \mathrm{~ns}, I_{3}(c)\right)$, and "o-Ps escape" model calculations (right) - annihilation lifetime in pores, $\tau_{f}(\mathrm{~d})$, escape rate and yield, $\kappa_{v}(\mathrm{e})$ and $\mathrm{Y}_{v}$ (f) - for $3 \mathrm{keV}$ positrons in mesoporous silica CTAB-TEOS S(x) film/glass for $\mathrm{CTAB} / \mathrm{Si}(\mathrm{x})$ mole fraction in the range $0-0.14$.

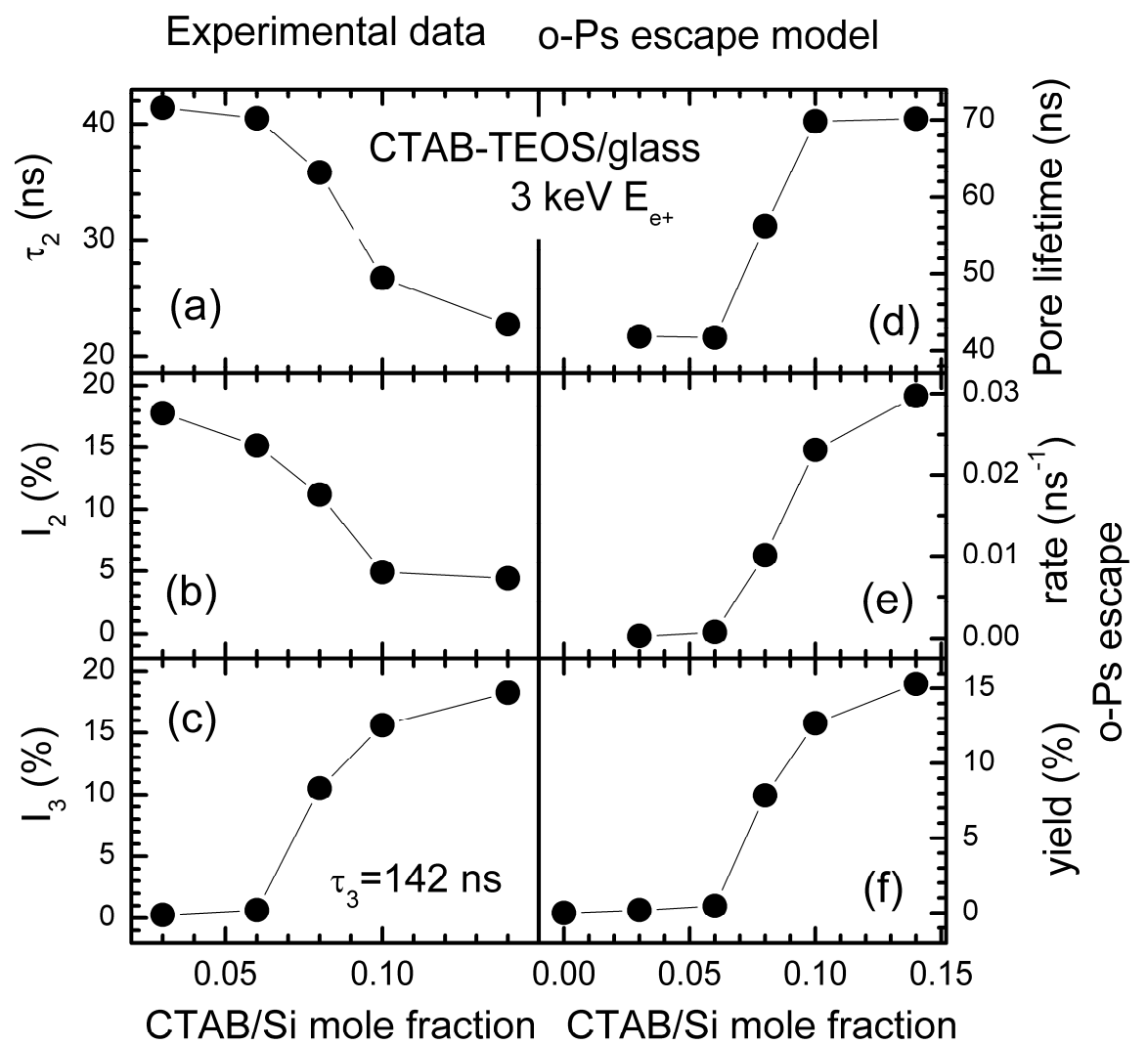


Figure 2.

Ortho-Positronium escape rate, $\kappa_{v}$, for $3 \mathrm{keV}$ positrons as a function of the mesopore volume fraction, $p_{m}$, in mesoporous silica $\mathrm{S}(\mathrm{x})$ films for $\mathrm{x} \geq 0.06$ and (insert) corresponding $o$ Ps escape yield, $\mathrm{Y}_{v}$, versus refractive index, $n$, measured at $633 \mathrm{~nm}$ wavelength.

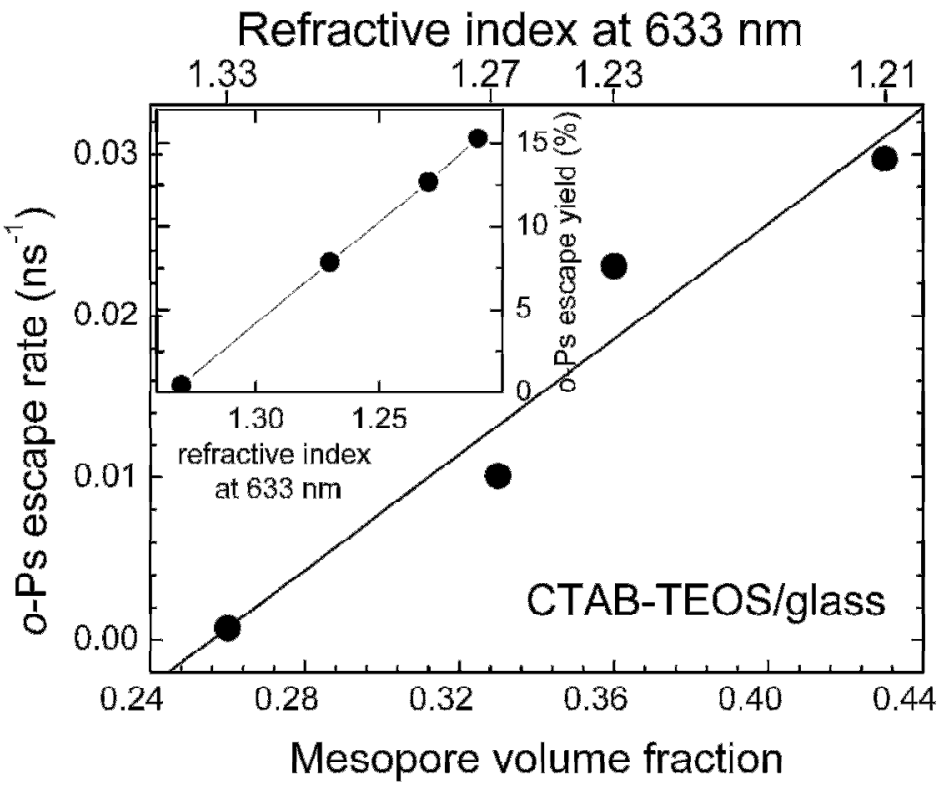

